REDES- Revista hispana para el análisis de redes sociales

Vol.15,\#7, Junio 2006

http: // revista-redes.rediris.es

\title{
Multitudes y Redes en la caida de Milosevic
}

\author{
Rodrigo Araya Dujisin - Departament d'Antropologia social i cultural (UAB) ${ }^{1}$
}

\section{Resumen}

El objetivo del estudio es analizar la composición y estructura de las redes personales de jóvenes serbios en relación a la masiva manifestación que se llevó a cabo el 5 de octubre de 2000 en la ciudad de Belgrado y que tuvo como resultado la caída del régimen de Slobodan Milosevic. El supuesto teórico que subyace en este estudio plantea que a nivel micro social podremos encontrar huellas y patrones del fenómeno macro social, en este caso de la Revolución de Octubre.

La revolución es analizada desde el concepto de cascada cooperativa, donde los comportamientos individuales se combinan para producir un fenómeno emergente. La cascada cooperativa se analiza desde la perspectiva de las redes personales, donde se combinan tres factores principales: disminución de los umbrales individuales de acción, características de los alteri (composición) y medidas estructurales de centralidad.

Palabras clave: redes personales, acción colectiva, caida de Milosevic.

\section{Abstract}

The aim of the study is to analyze the composition and structure of personal networks of young Serbs in relation to the massive demonstration that took place in Belgrade on October $5^{\text {th }}, 2000$ and whose main outcome was the fall of Milosevic's regime. The theoretical assumption is that at the micro level it is possible to find prints and patterns of the macro phenomena.

The revolution is analyzed from the concept of 'cooperative cascade', where individual actions are combined to produce an emergent phenomenon. The cooperative cascade is analyzed from the personal networks perspective, where three main factors are combined: decrease of individual thresholds, characteristics of alters (composition) and centrality measures (structure).

Key words: personal networks, collective action, fall of Milosevic.

\section{Perspectiva teórica}

El modelo teórico en el cuál se basa este estudio propone que la acción colectiva se produce como consecuencia de un proceso de "cascada informativa", donde aumentan explosivamente los flujos de información a través de las relaciones interpersonales. Una cascada de información es un evento durante el cual los individuos espontáneamente dejan de actuar como individuos y comienzan a cooperar, actuando como una masa coherente (Watts, 2003). Las cascadas informativas, ya sea en una situación de pánico financiero, turbas o revoluciones, pueden ser desencadenadas por un pequeño estímulo inicial y luego propagarse a través de un sistema completo. Es la idea de contagio social y reacción en cadena.

\footnotetext{
${ }^{1}$ Enviar correspondencia a: raraya@gmail.com
} 
La diferencia entre una cascada informativa y una cascada cooperativa radica en que en ésta última los individuos no sólo se dejan llevar sino que contribuyen decisivamente al movimiento social. Las cascadas cooperativas pueden ser definidas en un nivel micro social como la puesta en alerta y activación de toda la red personal de un individuo. Se pueden activar los lazos débiles e incluso los lazos latentes, aquellos que han estado inactivos por largo tiempo para asegurar el éxito de la movilización. A nivel macro social pueden ser observadas como estallidos masivos de cooperación donde se pasa de la lógica racional individualista (Olson) a una colectiva y del don (Mauss). Se ofrece la red y recursos personales para el logro de los objetivos colectivos.

De acuerdo a la teoría de Watts (2003) los grupos o redes con alta conectividad tienden a ser estables y presentan menor vulnerabilidad ante este tipo de fenómenos de contagio social y, por lo tanto, es menos probable que el origen de una cascada sea una red densa y de alta conectividad interna. Las cascadas a nivel de un sistema social pueden comenzar por dos vías, ya sea por la reducción del umbral promedio de la población o por la reducción de la densidad promedio de una red (cantidad de conexiones). Cuando ambos factores son altos (umbrales y densidad) el sistema tiende a ser estable. Para prevenir o precipitar una cascada global (a nivel de un sistema) la clave reside en no centrarse en el estímulo en sí mismo, sino en la estructura de la red en la cual el estímulo golpea (Watts, 2003).

\section{Ciclo de la acción colectiva}

El ciclo de la acción colectiva se puede describir desde la perspectiva de la difusión de informaciones. En este sentido la cascada cooperativa sería un episodio que sigue la curva de la difusión de informaciones de Rogers (2003).

En este caso, una primera fase se podría definir como individualismo racional, de la vida cotidiana, donde los comportamientos están regidos por la lógica de maximizar los intereses individuales. Una segunda fase se denomina agitación asociativa y se puede describir como la activación de las redes sociales por el aumento de los flujos de información y/o por ciertos cambios producidos en alguna parte sensible de la red, esto es, donde están los innovadores, adoptadores tempranos, quienes acogen determinada idea nueva que impacta en sus entornos inmediatos y luego en el resto de la red. Los individuos adoptan una innovación con la expectativa de que otros la adoptarán en el futuro. El efecto contagio ocurre cuando un individuo ve a sus pares adoptando una nueva idea. Observar mientras se es observado juega un papel especialmente importante en el proceso de difusión (Rogers, 2003) 
La agitación asociativa producida por la adopción de una nueva idea puede alcanzar el nivel de masa crítica, esto es, el umbral social o punto de quiebre (tipping point) en el proceso de difusión. Después de que la masa crítica es alcanzada, las normas del sistema social favorecen nuevas adopciones por parte de otros miembros del sistema. Cuando el nivel de masa crítica está cerca de ser alcanzado, basta con unos pocos nuevos adopta para marcar una gran diferencia, ya que la tasa de adopción rápidamente escala.

En el caso de la difusión de informaciones, el umbral (individual) se alcanza cuando un individuo está convencido de adoptar una idea nueva, al saber que cierto número de otros individuos en su entorno la han adoptado y están satisfechos con la innovación. Granovetter (1978) define umbral como el número de otros individuos que deberían estar vinculados a una actividad antes de que un individuo dado se vincule a tal actividad. Propone el siguiente ejemplo para describir los umbrales individuales: I maginemos 100 personas reunidas en una plaza en una potencial situación de disturbio. Supongamos que sus umbrales de disturbio están distribuidos como sigue: Hay un individuo con umbral 0 , otro con umbral 1 , otro con umbral 2, y así sucesivamente hasta el último individuo con un umbral 99. Ésta es una distribución de umbrales. El resultado es claro y puede ser descrito como efecto "subirse al carro" o "efecto dominó". La persona con umbral 0, el instigador, comienza el disturbio, rompe una ventana o lanza una piedra. Esto activa a la persona con umbral 1. La actividad de estas dos personas activa entonces a la persona con umbral 2 y así sucesivamente hasta que las 100 personas se han unido al disturbio.

El umbral de adopción ocurre a nivel individual, mientras que la masa crítica opera a nivel del sistema. Los individuos tienen umbrales de adopción, mientras que los sistemas sociales, comunidades y organizaciones tienen masa crítica. Una vez alcanzado el nivel de masa crítica el sistema entra en el episodio distribuido o cascada cooperativa, donde aumenta la frecuencia de las comunicaciones, se activan lazos débiles, latentes e incluso se superan trabas de distancia social y jerarquía. Los individuos dejan de actuar como individuos y son parte de una masa coherente, es decir, se ha producido la acción colectiva.

Luego del peak en el ciclo de la acción colectiva el sistema tiende a normalizarse. Se vuelve a los múltiples centros, se vuelve a un estado de agitación asociativa, disminuyen los flujos de información y finalmente se vuelve al estado de individualismo racional. No obstante la curva sugiere la idea de declive simétrico, esto es, volver al mismo punto de partida, es plausible sostener que no es así. La 
idea del 'efecto memoria' (Lozano, Borge, Arenas, 2007) sostiene que una vez que desaparece el estímulo que generó la transformación del sistema, éste no vuelve al mismo punto de partida. Hay un aumento de la cohesión del sistema después de una crisis y se observa un efecto memoria en el periodo entre crisis.

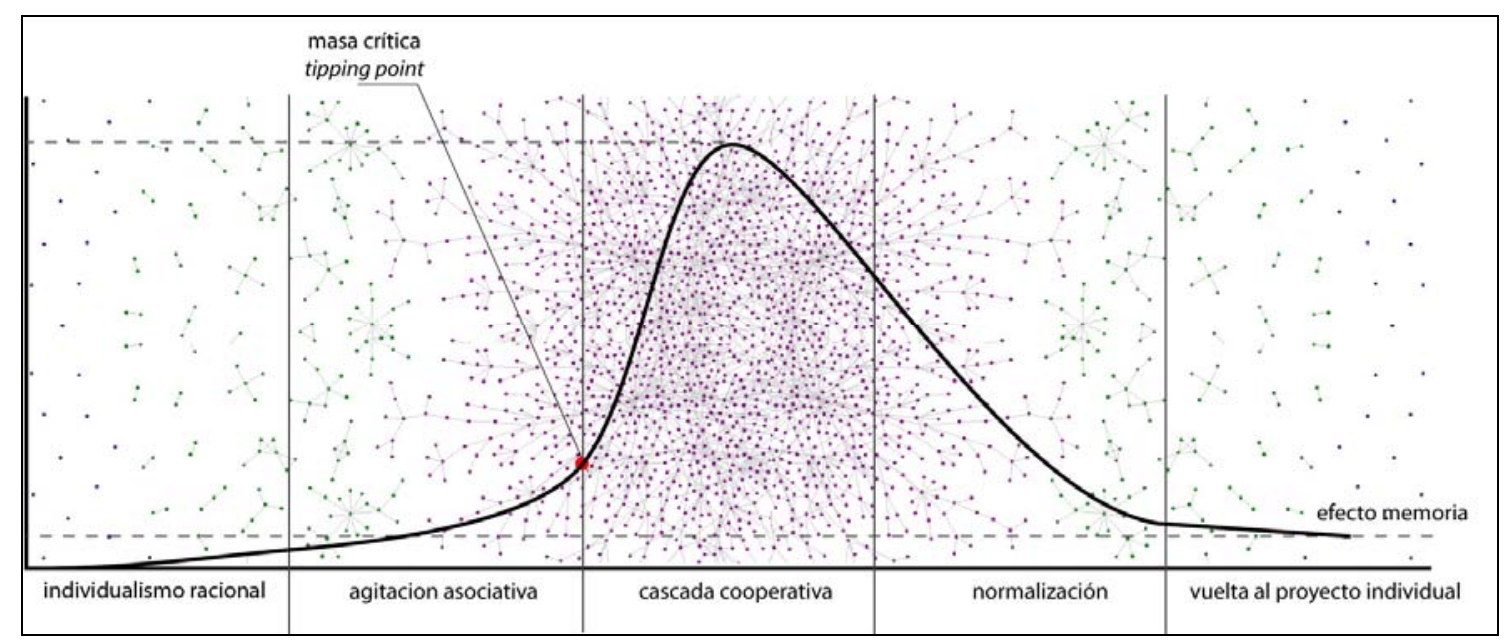

I lustración 1. Ciclo de la acción colectiva

\section{Diseño del estudio}

El estudio consistió en un análisis comparativo de la composición y estructura de las redes personales de jóvenes belgradenses. La estrategia para abordar este estudio consideró recorrer las redes de personas que participaron en la Revolución de Octubre. La muestra de casos se confeccionó con el procedimiento 'bola de nieve', esto es, recorriendo los contactos que los propios entrevistados van generando. De este modo se va recorriendo el 'mundo social' de los entrevistados primarios. Tambien se entrevistó a personas que no participaron de la manifestación para tener un parámetro de comparación de las redes personales.

Todas las personas entrevistadas son residentes en la ciudad de Belgrado al momento de la entrevista (entre noviembre de 2006 y mayo de 2007). La mayoría de las entrevistas ( 54 de 68 ) se hicieron a partir de dos casos o egos primarios. Por lo tanto, la mayoría de las redes personales están relacionadas a uno de los dos egos primarios. No es una muestra estadísticamente representativa, pero cualitativamente valiosa en tanto recorre y de alguna forma reconstruye un fragmento de la multitud.

Se realizó un levantamiento de información en distintos niveles. Se aplicó una encuesta a 100 personas y dentro de éstas se realizó una entrevista en profundidad con 68 casos. Cada una de las 68 entrevistas contiene información de 30 personas 
(alteri) con las cuales cada caso (ego) se relacionaba en el año 2000. En consecuencia, este levantamiento contiene información sobre 2040 personas (68 casos $\times 30$ alteri cada uno).

La mayoría de los estudios sobre redes personales se centran en los lazos fuertes, esto es, el núcleo central de la red que generalmente es el que provee apoyo social (Maya y Holgado, 2005). En este caso se han considerado los criterios propuestos por McCarty (2002) respecto a la estructura de las redes personales. McCarty plantea que a partir de 40 alteri se puede reflejar la estructura de la red personal en todas sus dimensiones. De esta forma, el estudio no se limita a los lazos fuertes del entrevistado, obteniendo un acercamiento más válido de la red personal.

En las aplicaciones piloto del cuestionario se trabajó con 45 alteri, sin embargo, resultó ser una cifra muy alta y difícil de completar por el entrevistado, ya que en este estudio se están analizando redes personales de un periodo pasado, lo que supone dificultades para recordar y reconstruir el mundo social de manera retroactiva. Además de lo anterior, las entrevistas con 45 alteri pueden durar más de una hora y media y aumenta el riesgo de deserción del entrevistado en mitad del proceso. Por otra parte, en el estudio 'Los puentes interlocales' (Araya y Maya, 2005) las redes con 25 alteri resultaron adecuadas para analizar la estructura, composición y funcionalidad de las redes personales de estudiantes universitarios en Sevilla. De este modo, se llegó a la conclusión de trabajar con 30 alteri para el estudio en Belgrado.

\section{Efecto memoria y sesgos}

Las entrevistas comenzaban con una conversación abierta para situar a la persona en el período de la revolución. Se le preguntaba sobre cómo era su vida en ese periodo, sus actividades principales, ya sea trabajo, colegio o universidad y cuáles eran sus rutinas cotidianas. La idea era situar a la persona en el contexto particular de ese periodo. Luego se le preguntaba por el día de la manifestación y se le pedía que recordara como comenzó aquel día, con qué personas estuvo, si recuerda la forma en que estaba vestido/a.

Esta primera fase de la entrevista era fundamental, ya que había que transportar a la persona a la época de la Revolución. El hecho de que el día 5 de Octubre sea un hito para el país y para muchas personas, ayuda a que los sucesos se recuerden con facilidad, ya que no es un día cualquiera al que se les está pidiendo que se transporten. Fue un momento único y para muchas personas está asociado a fuertes emociones. Algo equivalente sucedería si se realizara un estudio que intente 
reconstruir detalles cotidianos del 11 de septiembre del 2001 en Nueva York, o el 11 de marzo de 2004 en Madrid. Probablemente cualquier ciudadano berlinés recordará más detalles del 9 de noviembre de 1989 que de cualquier otro día de ese año. Son fechas especiales que la memoria registra de manera particular. Por lo tanto, en este caso el problema de la fiabilidad de la memoria es menos complicado que en otros estudios retrospectivos.

No obstante lo anterior, una cosa es la capacidad de recordar y otra cosa es qué se recuerda. Los estudios que abordan temáticas retroactivas enfrentan además del problema de la memoria, el problema de los sesgos. La memoria es selectiva (Molina, 2005) y a veces distorsiona aquellos elementos no deseados, o se adornan situaciones porque no se quiere revelar comportamientos, actitudes que ahora son indeseables.

En el estudio de Opp y Gern (1993) sobre las manifestaciones en Leipzig que derivaron en la caída del muro de Berlín en 1989, se descubrió que mucha gente negaba su militancia en el partido socialista de la RDA, al mismo tiempo que se sobreestimaba la participación que tuvieron en las manifestaciones de 1989. Este estudio fue realizado 4 años después de aquellas manifestaciones y plantea la importancia de considerar los sesgos en este tipo de análisis. Opp y Gern identificaron dos problemas que pueden distorsionar la fiabilidad de las respuestas en este tipo de estudios, lo que denominan el "efecto resultado" y el "efecto héroe". El primero se refiere a la tendencia de las personas a ajustar su cognición del evento a sus resultados, en este caso una revolución exitosa. El segundo sesgo, el "efecto héroe", se refiere a la tendencia a sobreestimar el rol que tuvieron en la revolución y los riesgos personales que corrieron, debido a lo bien visto y celebrado de los sucesos.

Algunas personas pueden considerar que participaron de la manifestación, aunque solo fueron a la esquina a comprar algo y vieron a la multitud pasar con sus propios ojos. Para controlar este sesgo se elaboró una pregunta que consideraba el incentivo moral a ser parte de la revolución. Ante la pregunta del cuestionario ¿participaste de la manifestación del 5 de Octubre? Se ofrecieron tres respuestas posibles. Además de las respuestas afirmativa y negativa se elaboró una respuesta intermedia que es 'no, pero me hubiera gustado estar'. Aquí caben todas las excusas posibles. Algunos señalaron que ese día no estaban en la ciudad, otros que estaban enfermos, etc. Pero deja la posibilidad de no ser un "anti-revolución" en la respuesta. 


\section{Procesamiento y análisis de la información}

A partir del cuestionario de 10 preguntas se elaboró una base de datos en SPSS de 100 casos, con más de 30 variables. Por otro lado, se construyeron 68 matrices de redes personales (de $30 \times 30$ actores) que se refieren a 2040 relaciones en total ( 68 egos x 30 alteri). Cada una de estas matrices fue manejada y analizada con UCInet (Borgatti, Everett \& Freeman, 2002) y la información resumen de cada uno de los 68 casos fue introducida posteriormente en SPSS junto con el resto de datos descriptivos. Posteriormente se eliminaron los casos del cuestionario que no tenían la red personal. Así, la base de datos en SPSS quedó con 68 casos y por cada uno de ellos una base de datos en UCInet. Para la visualización de las redes se utilizó el software Netdraw, un subprograma parte de UCInet.

\section{Cuestionario}

La entrevista/cuestionario consta de cuatro partes y tiene una duración de aplicación promedio de 1 hora. Las tres primeras partes son estructuradas y se realiza con el apoyo de un ordenador. La cuarta parte son observaciones cualitativas que surgen de la conversación.

Modulo 1: Perfil del entrevistado (ego)

El primer módulo es el perfil del entrevistado, en adelante 'ego'. En esta sección hay cinco preguntas de información básica del entrevistado. Nombre, edad, educación, estado civil, información de contacto. También se pregunta por variables descriptivas como acceso a tecnologías de información (teléfono móvil e Internet) en el año 2000 y en el año 2007. Participación en organizaciones sociales (2000 y 2007) y participación en la manifestación del 5 de octubre del año 2000.

Este primer módulo es una encuesta estructurada y se aplicó a 100 personas, de las cuales se logró hacer la red personal a 68 casos. Las 32 encuestas restantes fueron eliminadas del análisis.

Módulo 2: Generador de nombres

El objetivo de este módulo es obtener una lista de 30 personas con las que el entrevistado tenía algún tipo de relación en el año 2000. La idea es recrear la vida social de la persona en ese periodo. Si el entrevistado no recuerda un nombre o por alguna razón prefiere mantener los nombres en privado se utilizaron sobrenombres o etiquetas genéricas (el hermano de la vecina, etc.). 
La pregunta generadora de nombres es: a continuación señálame el nombre de personas con las que tenías algún tipo de relación en ese tiempo. Trata de recordar cómo era tu vida cotidiana, tus actividades principales y de acuerdo a eso me vas señalando los nombres. Pueden ser personas de la familia, colegio, vecindario, universidad, amigos de fiesta, etc. Pueden ser personas muy cercanas y otras no tanto, como un contacto de la universidad o un vecino a quien apenas saludabas cada mañana. También puedes mencionar personas con las cuales había una mala relación o alguien que falleció posteriormente.

La lista de nombres se han ingresado al ordenador a medida que se van mencionando, por lo tanto se tiene una lista de 30 nombre. Las personas nombradas son los 'alteri'. A continuación se realizan algunas preguntas básicas sobre cada alter. En sucesivas columnas se indica mediante códigos $(0,1)$ preestablecidos a) género de cada alter; b) si la persona estuvo en las manifestaciones del 5 de octubre. Si el entrevistado no sabe con seguridad o no recuerda, se le pide que señale su percepción al respecto (si cree que participó o no) de acuerdo al conocimiento que tiene de la respectiva persona. c) la tercera pregunta sobre cada alter es acerca de la influencia percibida para participar en los eventos. El objetivo es identificar a aquellos alteri que eventualmente influenciaron a ego para participar en las manifestaciones. La influencia es entendida de una manera flexible (opinión respetada, admiración, o simplemente porque cierto alter invitó a ego ese día para ir a las manifestaciones. d) la última pregunta de esta sección es sobre la influencia ejercida por ego en los alteri. Aquí el objetivo es identificar a las personas que (según la percepción de ego) recibieron su influencia para asistir a las manifestaciones. Se trata de personas invitadas por ego ese día o porque generalmente siguen sus opiniones/actitudes/comportamiento.

Como resultado de esta sección se obtiene una matriz donde están los nombres de 30 alteri y los atributos de cada uno definidos por las preguntas a, b, c y d.

Módulo 3: Matriz de adyacencia

En este modulo de la entrevista se le pregunta a ego por las relaciones entre cada alter de la lista. Se confecciona una matriz con los 30 nombres en las filas y en las columnas. Se comienza preguntando si el primer nombre en las columnas conoce a cada uno de los 29 nombre de la lista. La respuesta es sí cuando son amigos, familiares, compañeros de trabajo, conocidos, etc. Se considera una relación cuando dos personas se reconocen por el nombre y podrían detenerse en la calle, saludarse y eventualmente tomar un café. Las relaciones negativas también son 
consideradas. Nombre por nombre (alter por alter) se va preguntando por las relaciones de todos con todos.

Módulo 4: Observaciones sobre ego

En esta sección se escriben las notas, observaciones sobre cada ego que surgen durante la entrevista. La idea es describir a la persona. Generalmente la entrevista termina con una conversación abierta donde surgen temas interesantes de documentar, tal como situación socioeconómica, características de personalidad (sociable, tímido, etc.) y anécdotas sobre la Revolución de Octubre.

\section{Resultados}

El análisis de resultados ha seguido cuatro pasos consecutivos. En primer lugar se analiza el perfil de los entrevistados y se clasifican de acuerdo a su participación en la Revolución de Octubre. En segundo lugar se realiza un análisis de la composición de las redes personales, de acuerdo a los subgrupos identificados en la etapa anterior. En tercer lugar se analiza la estructura de las redes personales, de acuerdo a las medidas de centralidad de Freeman (1978). Finalmente se propone una tipología de las redes personales.

\section{Perfil de los entrevistados}

Los entrevistados tienen en promedio 30,1 años de edad, siendo 21 años la mínima y 59 la edad máxima de los casos entrevistados. El 67,6\% de los casos se concentra en el tramo de edad que va de los 21 a los 29 años. Un 16\% está en el tramo que va entre los 30 y 39 años y otro $16 \%$ está en el tramo que va de los 40 a los 59 años de edad.

Una amplia mayoría de los casos son solteros (82\%), mientras un $15 \%$ están casados y sólo un 2,9\% son divorciados. La mayoría de las personas entrevistadas (88\%) reside con su familia, mientras unos pocos viven con su pareja (7\%) y quienes viven en un piso compartido o sólo resultaron ser casos muy minoritarios ( $1,5 \%$ cada uno).

Para el año 2000 más de un tercio de los entrevistados (38\%) estaba en el colegio y una proporción similar (35\%) eran estudiantes universitarios. Dentro de éstos últimos un $10 \%$ trabajaba además de estudiar. De los casos restantes casi un cuarto de la muestra (23\%) trabajaban en el año 2000 y una pequeña proporción (3\%) se encontraba desempleado. Al momento de la entrevista (2007) la mayoría 
de los casos (66\%) es estudiante universitario y algunos de ellos trabajan paralelamente.

Hasta aquí tenemos variables descriptivas de los entrevistados que se pueden resumir en que la mayoría son jóvenes, solteros y para el año 2000 una amplia mayoría vivía con sus familias y su actividad principal era la de estudiante, ya sea del colegio o universidad.

\section{Clasificación de los casos}

Un segundo paso en el análisis consiste en clasificar a los entrevistados de acuerdo a la variable que haría de filtro al conjunto de la muestra. De este modo, los casos se clasificaron entre quienes participaron de la Revolución, quienes no participaron y quienes no lo hicieron, pero les habría gustado. Esta tercera alternativa se estableció para minimizar el impacto del 'efecto resultado' y el 'efecto héroe' que describieran Opp y Gern (1993) para el caso de Alemania del Este. Dado que el evento en cuestión fue exitoso y está asociado a una serie de atributos social y moralmente deseables, se estableció como alternativa para atraer a aquellos que podrían verse tentados con el efecto resultado y el efecto héroe.

Del total de entrevistados (68) una mayoría clara (56\%, equivalente a 38 casos) señala haber participado de la Revolución manifestándose en la calle aquel día 5 de octubre. Un segundo grupo de entrevistados (29\%, equivalente a 20 casos) son aquellos que no participaron del evento, ya sea por no compartir los objetivos políticos o los métodos de la acción colectiva. El tercer grupo lo conforman aquellos que no participaron, pero les habría gustado estar ahí (14\%, el equivalente a 10 casos). En adelante a estos grupos le llamaremos los revolucionarios, conservadores y efecto héroe, respectivamente.

\section{Tecnologías de información y membresía en organizaciones}

Dos hipótesis complementarias fueron puestas a prueba en la encuesta. La primera hipótesis es sobre el rol que eventualmente tuvieron las tecnologías de la información en la coordinación de acciones durante la revuelta de Octubre. A pesar de que para el año 2000 las tasas de penetración de internet eran bastante bajas a nivel mundial, se ha planteado en algunos documentos no académicos (documentales, prensa y blogs) que éstas tuvieron un rol importante en la manifestación. 
Para el año 2000 la penetración de Internet en Europa era del 14\%. En Serbia y Montenegro para ese año la tasa era de un $4 \%$ de la población (ITU, 2003). No obstante, esta cifra es a nivel nacional e incluye a Kosovo, donde la penetración era casi inexistente para esa fecha. Por lo tanto, si se considera Serbia sin Kosovo la tasa de penetración sube a $7 \%$ y si se considera solo Belgrado la tasa alcanza el 18\% para el año 2000 (Belgrade Open School, 2007). Por último, si la tasa de penetración se analiza por tramo de edad probablemente la tasa aumente significativamente. Está ampliamente documentado que en las primeras fases de la masificación de internet los usuarios eran mayoritariamente jóvenes entre 15 y 29 años. No hay cifras de penetración por tramo de edad para el año 2000, pero una proyección razonable podría establecer que el 35\% de los belgradenses menores de 30 años eran usuarios de Internet en el año $2000^{2}$.

Al momento del estudio $(2006,2007)$ casi la totalidad de los entrevistados tenía acceso a alguna tecnología de información, ya sea sólo a móvil (17\%) o móvil e Internet $(81 \%)$. Ahora bien, lo que interesa es saber cómo era la situación en el año 2000. De la muestra consultada en este estudio un $41 \%$ señala no tener ningún tipo de acceso a tecnologías de información en el año 2000, ya sea Internet o teléfono móvil. Un $16 \%$ señala tener acceso sólo a teléfono móvil, mientras un $17,6 \%$ sólo a Internet. Por último, un $25 \%$ de los consultados señala tener acceso a móvil e Internet en el año 2000 . Por lo tanto, un $42,6 \%$ de los casos tenía acceso a Internet (sumando sólo Internet y móvil más Internet) y un 59\% a algún tipo de tecnología de información (sólo móvil, sólo Internet o ambos).

Si consideramos que el $67,6 \%$ de los casos de este estudio tienen entre 21 y 29 años al momento de la entrevista, es decir, tenían entre 15 y 23 años en el año 2000, las altas cifras no deberían sorprender mayormente, ya que es un segmento muy específico de la población que se caracteriza por la adopción temprana de estas tecnologías.

Ahora, si analizamos el acceso a las tecnologías según los grupos establecidos (revolucionarios, conservadores y efecto héroe) los resultados son sugerentes, ya que el $50 \%$ de los revolucionarios tenía acceso a Internet en el año 2000, mientras los conservadores sólo el $30 \%$. No obstante, estas cifras no dan para entusiasmarse demasiado, ya que la muestra es muy pequeña y la dispersión alta,

\footnotetext{
2 Esta proyección es propia y está basada en los reportes de ITU (Internacional Telecomunication Union). La cifra propuesta (35\%) se basa en datos desagregadas por tramo de edad en paises con tasas de penetración similares a la de Serbia y Montenegro para el año 2000.
} 
por lo que unos pocos casos pueden significar una gran variación. Si bien no son cifras estadísticamente significativas, si las podemos considerar sugerentes para caracterizar al grupo de los revolucionarios.

Respecto a la membresía en organizaciones formales, también se encuentran ligeras tendencias entre los grupos analizados. Mientras el $72 \%$ de los revolucionarios ha participado en algún tipo de organización formal (juvenil, política, deportiva, social), solo el $25 \%$ de los conservadores lo ha hecho. Nuevamente hay que destacar que estas cifras hay que valorarlas con precaución. Se trata de señales sugerentes a partir de una muestra muy pequeña.

\section{Composición de la red}

Una de las hipótesis centrales de este estudio sostiene que a nivel microsocial podremos encontrar huellas del fenómeno macrosocial, en este caso la Revolución de Belgrado. Una segunda hipótesis propone que las redes personales fueron el principal canal de difusión de información e influencia mutua para participar de la manifestación, esto es, la masa crítica se logró a nivel micro, rompiendo los umbrales de participación a nivel interpersonal. Dicho de otro modo, una vez alcanzado cierto umbral de adopción de una conducta, es muy probable que se adopte aquel comportamiento. Esta hipótesis está ampliamente analizada para los procesos de difusión de innovaciones (Rogers, 2003; Valente, 1996).

La proposición de los umbrales en las redes sociales para la difusión de innovaciones se basa en el principio de la homofilia (Rogers, 2003) que básicamente consiste en que el intercambio de ideas ocurre con mayor frecuencia entre iguales, ya que la comunicación es más efectiva cuando emisor y receptor son parecidos. Homofilia es el grado de similitud entre dos individuos que se comunican y es un acelerador de las innovaciones, pero dentro de los límites de la red de iguales. La heterofilia, en cambio, es el grado de diferencia entre individuos que se comunican y tiene la propiedad de conectar mundos distintos. Los vínculos interpersonales heterófilos en un sistema tienen la propiedad de hacer puente, lo que es especialmente importante para la transmisión de nuevas ideas, innovaciones, tal como lo plantea la teoría de la fuerza de los lazos débiles de Granovetter (1973). Es decir, las relaciones heterófilas son claves en la adopción de nuevas ideas y la homofilia es el acelerador de la difusión de determinada innovación o comportamiento. Las cascadas de información y cooperación, en consecuencia, se producen cuando ciertos umbrales de adopción son superados y la nueva idea, información o comportamiento se difunde a través de redes de iguales. 
Para poner a prueba esta hipótesis se analiza la composición de las redes personales de los 68 egos y sus 2040 relaciones. Para ello se calculó el número promedio de personas que participaron de la Revolución en cada red personal. Los resultados señalan que los revolucionarios tienen en promedio 16 alteri que participaron de la manifestación, en cambio los conservadores 8,6. Dicho de otro modo, los revolucionarios tienen en promedio 7,4 revolucionarios más en su red que los conservadores. Aquellos clasificados como 'efecto héroe' tienen en promedio 13 alteri que participaron de la revolución.

En consecuencia, los revolucionarios tienen más revolucionarios en su red personal y los conservadores, tienen más conservadores. Es decir, superado cierto umbral de alteri que participaron o no en la revolución, podríamos predecir con una alta probabilidad si determinado ego es revolucionario o conservador.

Resulta interesante el caso de los 'héroes', cuyas redes personales tienden a parecerse a la de los revolucionarios (el comportamiento deseado), aunque con menor número de personas que participaron de la revolución.

\begin{tabular}{|c|c|c|}
\hline PARTICI PACI ÓN EN LA MANI FESTACI ÓN & & ALTER SI* \\
\hline SI & Media & $\mathbf{1 6}$ \\
\hline & $\mathrm{N}$ & 38 \\
\hline & Desv. típ. & 5,685 \\
\hline NO, pero me habría gustado estar & Media & $\mathbf{1 3 , 3}$ \\
\hline & $\mathrm{N}$ & 10 \\
\hline NO & Desv. típ. & 5,293 \\
\hline & Media & $\mathbf{8 , 6}$ \\
\hline & $\mathrm{N}$ & 20 \\
\hline Total & Desv. típ. & 4,795 \\
\hline & Media & $\mathbf{1 3 , 4 3}$ \\
\hline & $\mathbf{N}$ & $\mathbf{6 8}$ \\
\hline & Desv. típ. & $\mathbf{6 , 2 3 3}$ \\
\hline
\end{tabular}

* La variable Alter si corresponde al número de alteri que participaron en la revolución.

Tabla 1. Composición de las redes personales

La otra variable analizada respecto a la composición de las redes personales es la de influencia recibida y ejercida en otros. En el módulo 2 del cuestionario (generador de nombres) preguntábamos por la influencia que ego recibió de sus alteri, así como la influencia que eventualmente ego tuvo en ellos. Se calculó el número total de alteri influenciadores e influenciados para comparar la composición de las redes. 
Los resultados muestran que los revolucionarios en promedio fueron influenciados por 8 personas de su red personal, mientras que ellos influenciaron a otras 8,4 personas de su red para participar de la Revolución. Los conservadores, en cambio, recibieron influencia de 5,6 personas de su red para no participar de la revolución e influenciaron a otros 5,3 para resistir la cascada revolucionaria.

El resultado más sorprendente es el de los 'héroes', ya que señalan haber sido influenciados por 8,4 personas y declaran haber influenciado a otras 10,5 para participar de la revolución. Es decir, los que no participaron de la revolución pero les habría gustado, declaran haber sido más influyentes que aquellos que participaron activamente. Probablemente aquí estamos ante lo que Opp y Gern describieron como una tendencia a sobreestimar el rol que tuvieron en la revolución.

Dentro de los revolucionarios hay una percepción de mayor influencia recibida y ejercida en otros que entre los conservadores. Por cierto hay que considerar que los revolucionarios están imbuidos en un proceso de cascada cooperativa, mientras los conservadores tienen menos incentivos para ejercer influencia ya que la ola va en otra dirección.

Todo lo anterior hay que tomarlo como lo que es: una primera aproximación a un fenómeno social emergente, complejo, poco estudiado, pero que tuvo importantes consecuencias para la historia reciente del país.

\section{Estructura de la red}

Para analizar la estructura de las redes personales se han utilizado las medidas de centralidad, también conocidas como Medidas de centralidad de Freeman, dado su influyente trabajo 'Centrality in Social Networks' (Freeman, 1978). Se trata de cuatro medidas que se utilizan para caracterizar la estructura de las redes sociales. Centralidad, cercanía, intermediación (Freeman, 1978) y Eigenvector.

Estas cuatro medidas permiten caracterizar la estructura de las redes personales analizadas. Para cada entrevistado se calcularon con UCInet los indicadores de centralidad promedio de su red personal $(30 \times 30)$. Concretamente, se calculó el promedio de lazos de los actores de la red personal (degree); la cercanía promedio (closeness); el promedio de geodésicos (el camino más corto entre cada par) que pasan por cada actor (betweeness) y el indicador promedio de centralidad eigenvector. 
Los resultados del análisis muestran que las redes personales de los conservadores, en sus propiedades estructurales, tienen un mayor nivel de integración y densidad. Concretamente, muestran un índice promedio de centralidad de grado más elevado que las redes de los revolucionarios. Esto quiere decir que los conservadores tienen redes más densas y con mayor conectividad. La medida de cercanía muestra una tendencia similar, ya que entre las redes de los conservadores un nodo determinado está en promedio más cerca de los otros nodos de la red. Por el contrario, el promedio de intermediación es mayor en las redes personales de los revolucionarios. Esto quiere decir que en promedio estas redes tienen mayor capacidad de conectar partes distintas de la red.

Pese a tratarse de índices poco específicos de las propiedades estructurales de las redes personales (se trata de cálculos promedio), los datos apuntan a una diferente conformación de las relaciones en función de la participación en la revolución.

\begin{tabular}{|r|r|r|r|r|r|}
\hline $\begin{array}{c}\text { Participación } \\
\text { manifestación }\end{array}$ & & Degree & \multicolumn{1}{c|}{ Closeness } & Betweenn & \multicolumn{1}{c|}{ Eigenvec } \\
\hline SI & Media & 41,21821 & 55,03663 & 2,45353 & 21,85703 \\
\hline & $\mathrm{N}$ & 38 & 38 & 38 & 38 \\
\hline & Desv. típ. & 12,20267 & 17,072303 & 0,916324 & 6,773009 \\
\hline $\begin{array}{r}\text { NO, pero me habría } \\
\text { gustado estar }\end{array}$ & Media & 42,0459 & 57,2758 & 2,3784 & 23,1982 \\
\hline & $\mathrm{N}$ & 10 & 10 & 10 & 10 \\
\hline & Desv. típ. & 4,786025 & 13,37618 & 0,508213 & 0,974289 \\
\hline NO & Media & 50,6782 & 61,8687 & 1,9397 & 23,7853 \\
\hline & $\mathrm{N}$ & 20 & 20 & 20 & 20 \\
\hline & Desv. típ. & 14,62718 & 13,382912 & 0,76113 & 1,198839 \\
\hline Total & Media & 44,12228 & 57,37535 & 2,29135 & 22,6214 \\
\hline & $\mathrm{N}$ & 68 & 68 & 68 & 68 \\
\hline & Desv. típ. & 12,81555 & 15,649683 & 0,845947 & 5,162722 \\
\hline
\end{tabular}

Tabla 2. Medidas de centralidad promedio de las redes presonales

En suma, los revolucionarios tienen en promedio redes menos densas que los conservadores y con mayor grado de intermediación. Esto podría considerarse como un grado mayor de diversidad de la red. Por otro lado, las redes de los conservadores tienen un alto potencial para la difusión de alguna información o comportamiento, el cual circulará con mayor velocidad, aunque al ser redes más cerradas hay menos circulación de ideas nuevas.

Hasta aquí creemos haber ofrecido soporte empírico modesto, pero valioso, para la comprensión de un fenómeno social como es la manifestación que provocó la caída de Milosevic. Las condiciones históricas y políticas son necesarias, pero no 
suficientes. Es necesario además que se produzca un fenómeno de cascada, un cambio en los sentimientos de confianza, una ruptura de las instituciones que rigen la interacción cotidiana, para que el fenómeno tenga lugar. Después, este fenómeno no será puntual, sino que tendrá efectos de nuevo en la vida cotidiana de las personas por los cambios macro-institucionales que se produjeron.

\section{Tipología de redes personales}

A partir de la tipología establecida (revolucionarios, conservadores) se seleccionaron 8 casos de cada una de las categorías extremas para realizar un examen exploratorio de la representación gráfica de las redes personales. El principal criterio para la selección de los casos fue la composición de la red personal. Se eligieron los casos más representativos de cada grupo, procurando mantener la heterogeneidad interna en cada tipo ideal. De este modo, seleccionamos 8 casos de los 38 que corresponden a los revolucionarios $(21 \%$ del total) y 8 casos de los 20 conservadores ( $40 \%$ del total). Es importante señalar que las representaciones gráficas sugieren ideas para poner a prueba, sugerir nuevas hipótesis más que proponer reflexiones concluyentes.

Para cada caso se elaboró un gráfico de las relaciones entre los 30 alteri mencionados por cada encuestado. La composición de la red personal fue representada con diferentes colores: los nodos rojos corresponden a los revolucionarios, aquellos que asistieron a la manifestación, mientras que los nodos amarillos corresponde a los conservadores. Cada gráfico corresponde a la red personal de un ego (entrevistado), el cual no aparece en la representación, ya que distorsionaría la estructura apareciendo siempre en el centro. La forma de las redes y la distribución espacial de los nodos la establece el software de análisis (UCInet) basado en las medidas estructurales. Dicho explícitamente, esto no es un dibujo, sino una representación analítica.

Al analizar el conjunto de casos seleccionados para cada tipo ideal surgen las siguientes características de las redes personales.

\section{Los revolucionarios}

Son aquellos que tienen redes personales donde al menos dos tercios de su entorno (66\% o más) participaron en la manifestación del 5 de octubre. Son redes donde los umbrales de adopción se han superado, ya sea un umbral de adopción de innovaciones (Rogers, 2003), de una acción colectiva (Coleman, 1990) o un umbral de rebeldía (Urrutia, 2003). En otras palabras, el entorno es lo suficientemente 
claro, respecto al comportamiento analizado, como para que ego se sienta socialmente aceptado para adoptar tal comportamiento. Quienes tienen umbrales bajos son los innovadores y quienes tienen umbrales muy altos son los rezagados. En este estudio no conocemos el umbral particular de cada ego, pero si tenemos certeza de que ante un entorno mayoritariamente orientado a cierta conducta, alcanzada la masa crítica que desencadena las cascadas, la probabilidad es muy alta de que ego se suba a la ola y lo lleve la cascada.

Así, vemos que en las redes de los revolucionarios predominan las relaciones con otros revolucionarios. Se podría especular que la red está movilizada para la revolución. Hay flujo de información y coordinación de acciones.

Por otro lado, las redes muestran una estructura más heterogénea y se pueden ver distintos grupos. En algunos casos (C25, C23) hay grupos totalmente desconectados de otros. Esto nos indica un alto nivel de intermediación de ego en la red personal. Cuando hay grupos sin conectar en la representación, quiere decir que ego es el puente entre aquellos grupos, como por ejemplo los amigos del barrio y los amigos de la universidad.

Dentro de los revolucionarios se identificó un subgrupo con características particulares. Se trata de personas que a pesar de tener una red personal mayoritariamente conservadora, asistieron a la manifestación. Les denominaremos los 'innovadores', ya que son revolucionarios a pesar de sus entornos conservadores, tal como lo muestran los casos $\mathrm{C} 10$ y C34, donde prácticamente todos los nodos son amarillos. No obstante lo anterior, en ambos casos hay un revolucionario en el entorno cercano (en el centro de la red, en rojo) lo que podría indicar que se trata de una influencia para ego o un influenciado de ego, podría ser otro innovador o, al menos un adoptador temprano (Rogers, 2003)

\section{Los conservadores}

Al igual que en el caso de los revolucionarios se trata de redes donde más de dos tercios de su entorno presentan un comportamiento similar. Como se observa en las representaciones, las redes de los conservadores están compuestas mayoritariamente de otros conservadores (nodos amarillos), es decir, se comprueba nuevamente el principio de la homofilia en las relaciones sociales.

Por su parte, se trata de redes ligeramente más densas y apiñadas que las de los revolucionarios. No se observan componentes o grupos definidos y hay menor variabilidad en la estructura de la red. 
Como se señalaba anteriormente las redes densas tienen un alto potencial para la difusión de informaciones o comportamientos, ya que hay mayor conectividad, pero son más resistentes a la entrada de nuevas ideas, ya que son más cerradas.

\section{Efecto héroe}

Las redes de los que no estuvieron pero hubieran querido estar presentan una alta variación, presentando características de ambos tipos ideales. No se encontraron particularidades en la estructura de estas redes. Algunas son prácticamente iguales a las de los revolucionarios y otras a las de los conservadores, por lo tanto no se pueden sugerir conclusiones a partir del análisis de las representaciones. La importancia de aislar este grupo del análisis radica en que permite caracterizar a los conservadores no sólo por la composición y estructura de la red, sino además por la posición política que tienen. Excluidos lo que compartían los valores de la revolución a pesar de que no estuvieron, nos ayuda a caracterizar a los conservadores partidarios del régimen y opuestos a los cambios democráticos. 


\section{Revolucionarios}

C 15

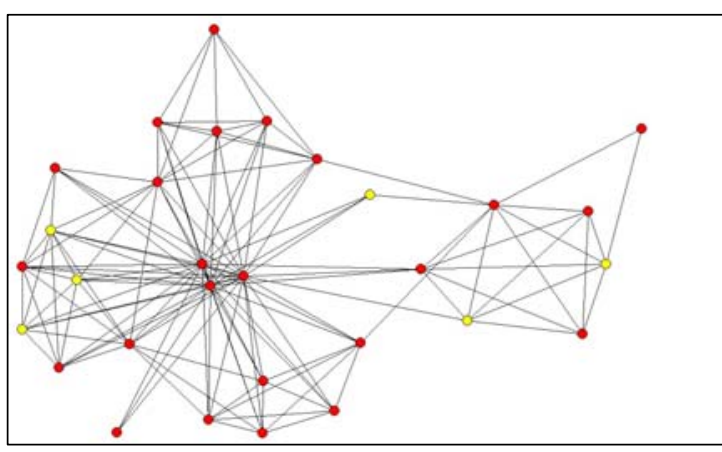

C 36
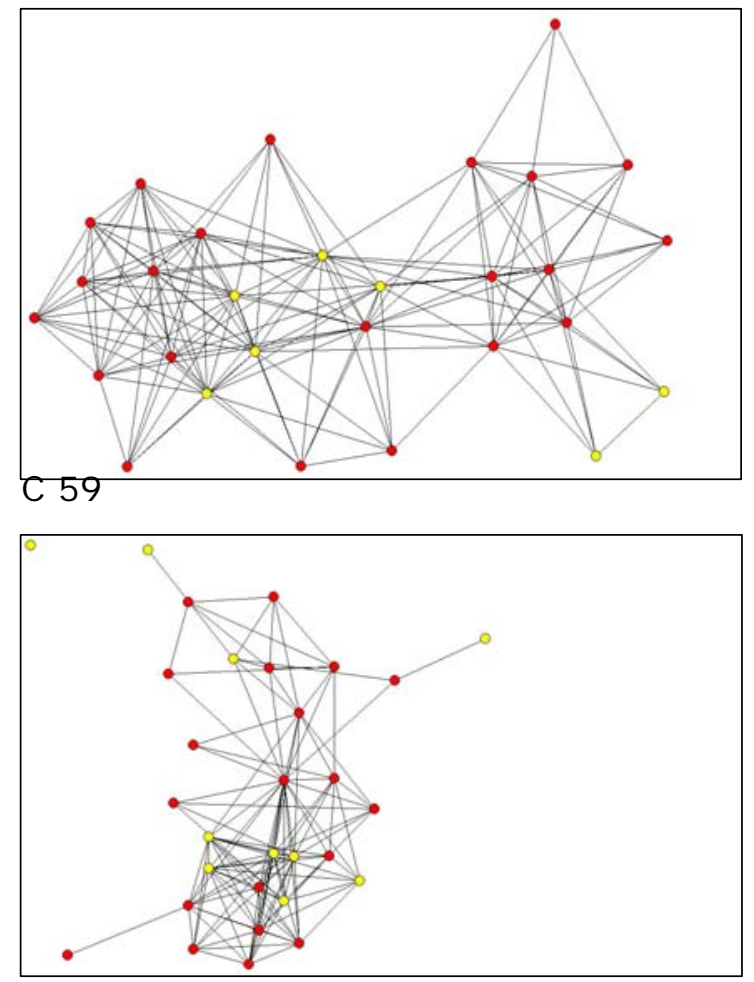

C 2

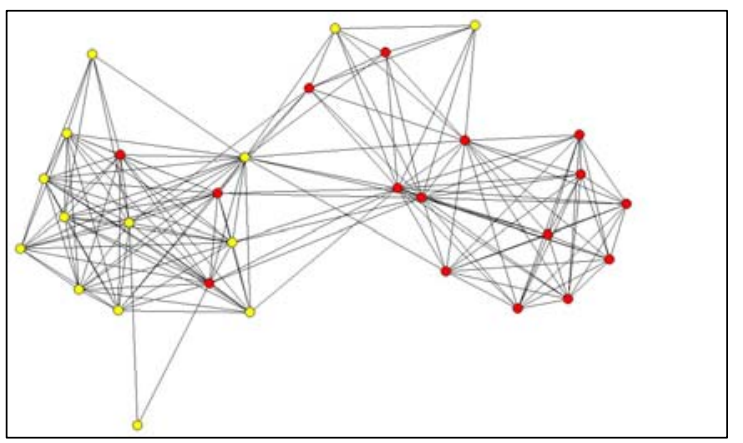

C 25

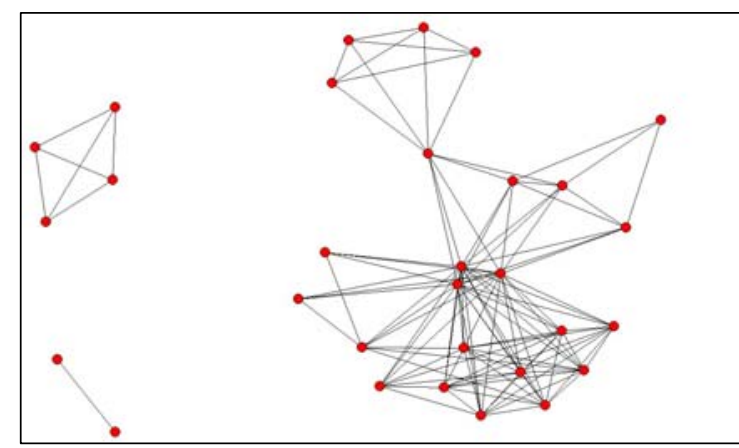

C 30
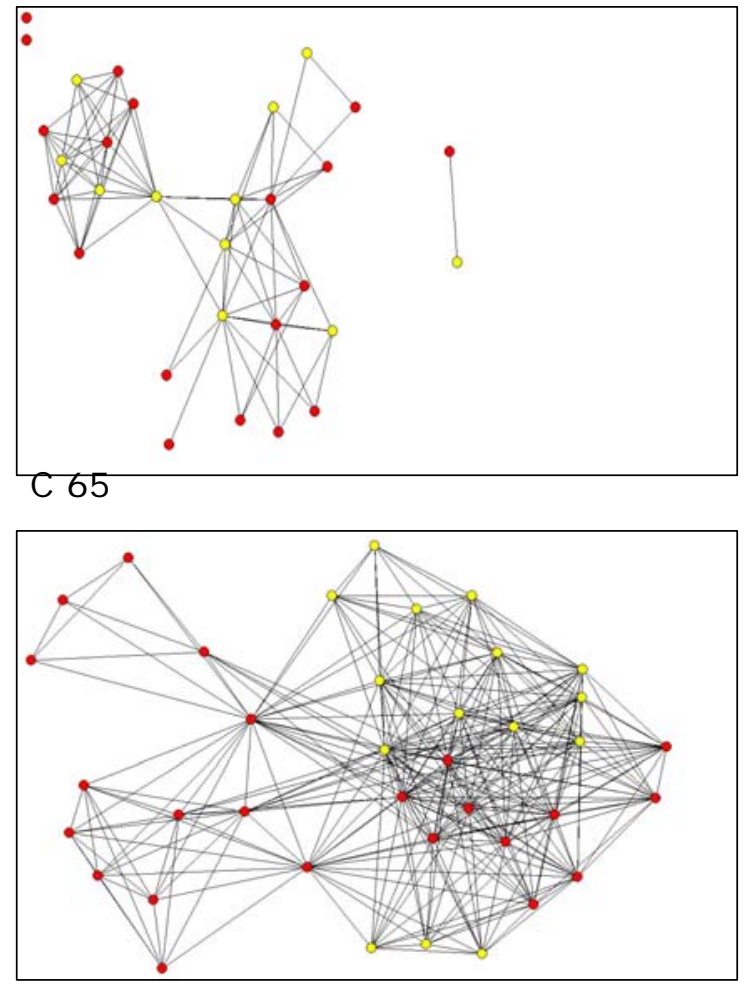

C 23

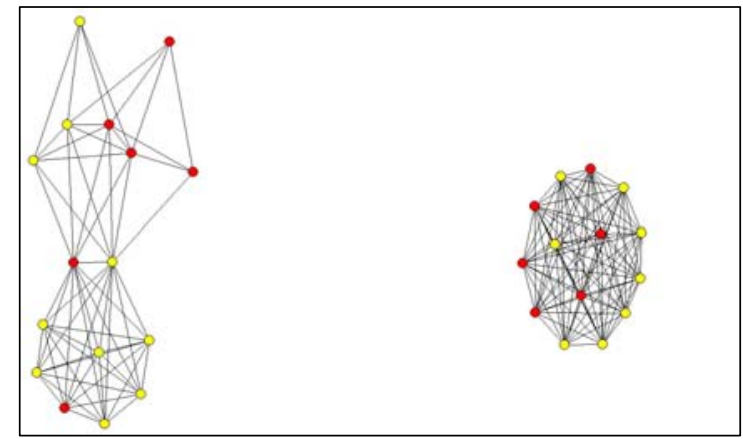




\section{Conservadores}

C 6

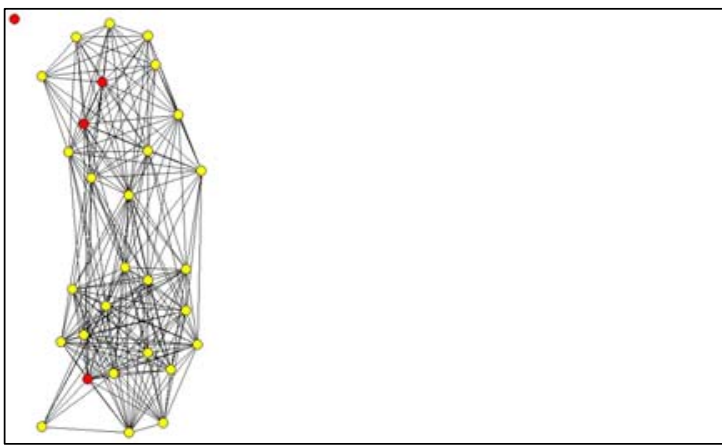

C 27

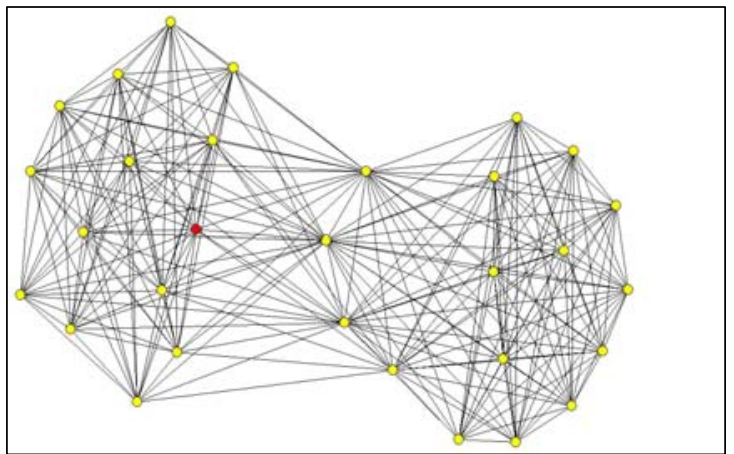

C 48

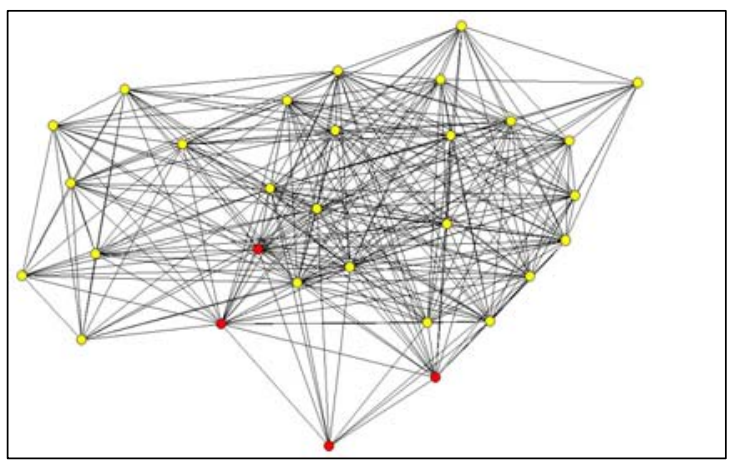

C 22

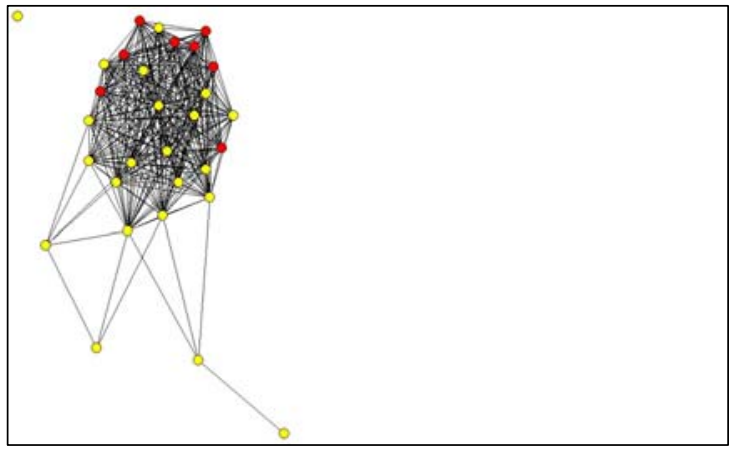

\section{26}

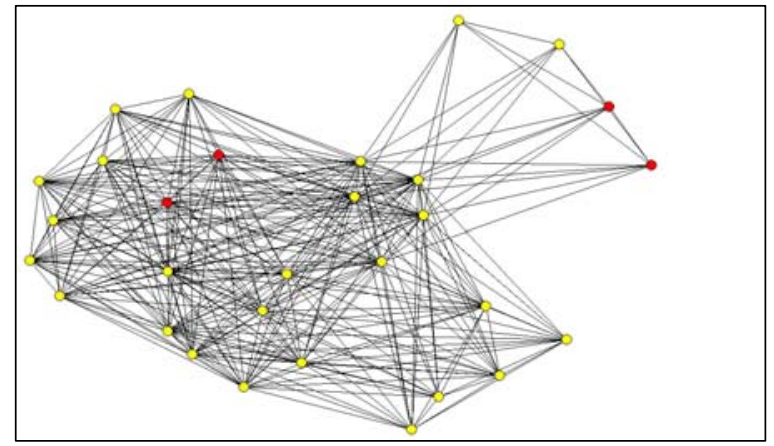

\section{45}

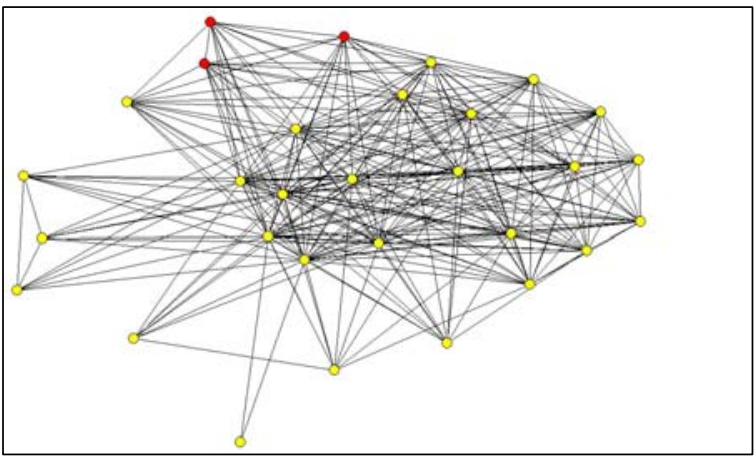

C 49

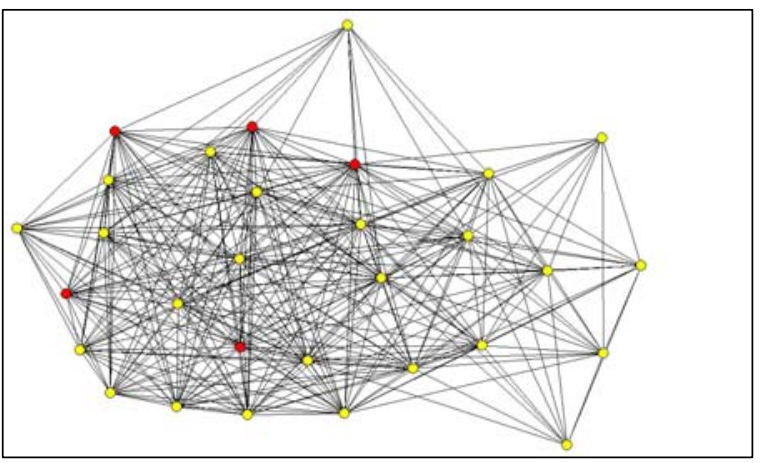

C 12

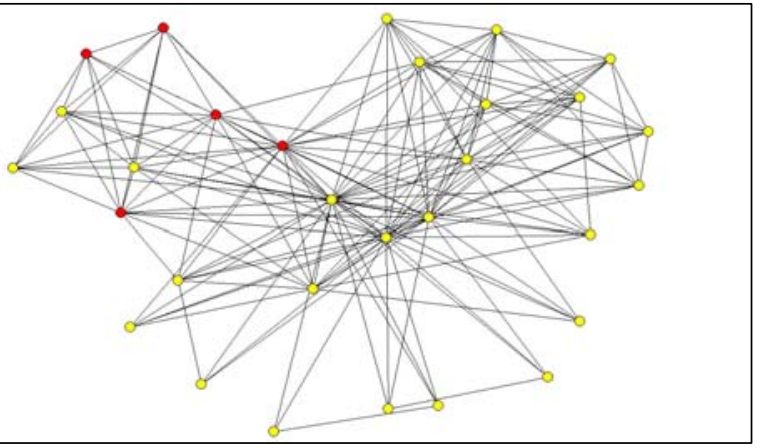




\section{Discusión}

La acción colectiva se produce desde acciones individuales y los actores evalúan permanentemente lo que hacen los otros para tomar sus propias decisiones. Los individuos hacen una transferencia unilateral de control sobre las acciones, y es esta transferencia la que constituye el núcleo a nivel micro de la explicación del comportamiento colectivo. De acuerdo a Coleman (1990) las personas intencionalmente hacen una transferencia de control de las acciones y este comportamiento individual se combina para producir el fenómeno a nivel macro, a veces espectacular y sorprendente, como las multitudes y revoluciones.

La aguda crisis política e institucional de Serbia (nivel macro) tuvo como consecuencia la disminución de los umbrales individuales de adopción (nivel micro) de las nuevas ideas y comportamientos necesarios para cambiar la situación. La disminución de los umbrales individuales favorece la transferencia de control de las acciones, generando las condiciones para que se produzca el comportamiento colectivo. La masa crítica, en consecuencia, se logró a nivel micro, rompiendo los umbrales de participación a nivel interpersonal.

El análisis de las redes personales muestra que quienes participaron en la revolución tienen características relativamente definidas (respecto a quienes no lo hicieron) en cuanto a composición y estructura. La composición de las redes personales de los 'revolucionarios' tienen en promedio 16 alteris revolucionarios (de un universo de 30), en cambio los conservadores solo 8,6. Asumiendo que los umbrales individuales han disminuido debido a la crisis aguda que vive el país, la adopción de las ideas revolucionarias se ve favorecida y reforzada por las propias redes interpersonales. La composición de las redes analizadas es consecuente con los postulados de los umbrales individuales de adopción (Granoveter, 1978; Watts, 2003).

Respecto a la estructura las redes personales, basándonos en las medidas estructurales de centralidad, observamos que los revolucionarios tienen redes menos densas y de mayor intermediación que los conservadores. Al ser redes menos densas, se hace posible la introducción de nuevas ideas, y al ser redes con mayor capacidad de puente (intermediación) se acelera el proceso de difusión entre distintas redes personales. Por su parte, las redes de los 'conservadores' muestran características de estabilidad en términos de adopción de innovaciones, lo que es congruente con la teoría de Watts. 
La formulación de Watts señala que independientemente del estímulo que golpea las redes, para el caso de Belgrado el mensaje del cambio o los discursos políticos, lo que resulta fundamental es la estructura de las redes donde este mensaje golpea. Los datos levantados en este estudio concuerdan con esta formulación, en tanto los revolucionarios tienen redes menos densas y con mayor intermediación, lo que de acuerdo a la literatura revisada favorece el proceso de difusión de informaciones y adopción de ciertos comportamientos. En suma, este estudio reafirma, a pesar de la limitación de los datos, que la estructura de las redes es una de las claves para explicar el fenómeno de cascadas cooperativas a nivel de un sistema.

\section{Bibliografía}

Araya Dujisin, R. y Maya Jariego, I. (2005). Los puentes interlocales: las redes personales de los universitarios alcalareños en Sevilla. En Redes, enfoques y aplicaciones del análisis de redes sociales. José I. Porras y Vicente Espinoza editores. IDEA.USACH - Universidad Bolivariana.

Belgrade Open School (2007). Internet penetration in Serbia 2006. Reporte con datos de Strategic Marketing Research.

Borgatti, S.P., Everett, M.G. y Freeman, L.C. Ucinet for Windows: Software for Social Network Analysis. Harvard: Analytic Technologies. 2002.

Freeman, L. C. (1978) 'Centrality in Social Networks. Conceptual clarification', Social Networks, 1 (1978/79) 215-239

Granovetter, Mark (1978). "Threshold Models of Collective Behavior", American Journal of Sociology, Vol. 83, No. 6, November 1978, pp 1420-1443

Maya Jariego, I. y Holgado, D.(2005) 'Lazos fuertes y proveedores múltiples de apoyo: comparación de dos formas de representación gráfica de las redes personales'. EMPIRIA. Revista de Metodología de Ciencias Sociales. № 10, pp 107127

ITU-International Telecomunication Union (2003), Yearbook of StatisticsChronological Time Series 1992 - 2001.

Mauss, Marcel. "Sociología y antropología”. Colección de Ciencias Sociales. Madrid. Editorial Tecnos, 1991. 
Mc Carty, C. "Structure in Personal Networks". Journal of Social Structure, №3. 2002

Molina, José Luis (2005). "El estudio de las redes personales: contribuciones, métodos y perspectivas", EMPIRIA. Revista de Metodología de Ciencias Sociales, № 10, pp 71-106

Olson, Mancur [1965] (1971). "The Logic of Collective Action: Public Goods and the Theory of Groups". Revised edition, Harvard University Press

Opp, Karl-Dieter; Gern, Christiane. (1993) “Dissident Groups, Personal Networks, and Spontaneous Cooperation: The East German Revolution of 1989", American Sociological Review, Vol. 58, No. 5, pp. 659-680

Rogers, Everett (2003). "Diffusion of Innovations". NY: Free Press.

Marian Borguñá, Romualdo Pastor-Satorras, Albert Díaz-Guilera, and Alex Arenas (2004). "Models of social networks based on social distance attachment", Fisical Review E 70, 056122

Urrutia, Juan (2003). '"Aburrimiento, Rebeldía y Ciberturbas: una aproximación a la economía Des-mercada". <http://juan.urrutiaelejalde.org/>

Valente, Thomas W. (1996). "Social network thresholds in the diffusion of innovations", Social Networks 18 (69-89).

Watts, Duncan J. (2003). "Six Degrees: The Science of a Connected Age". Cambridge; Cambridge University Press. 